\title{
Unresolved atelectasis in refractory Mycoplasma pneumoniae pneumonia: Predictive factors and the influence of corticosteroids and bronchoscopy lavage therapy
}

Jianhua Liu

Beijing Children's Hospital

Jinrong Liu

Beijing Children's Hospital

Bei Wang

Beijing Children's Hospital

Yajing Liu

Maternal and Child Health Care Hospital of Shunyi District

Chengsong Zhao

Beijing Children's Hospital

Fei Zhao

Chinese Center for Disease Control and Prevention

Jianzhong Zhang

Chinese Center for Disease Control and Prevention

Shunying Zhao ( $\sim$ zhaoshunying2001@126.com )

Beijing Children's Hospital

Research article

Keywords: mycoplasma pneumoniae; pneumonia; atelectasis; C-reactive protein; lactate dehydrogenase; bronchoscopy lavage therapy; Corticosteroid

Posted Date: March 9th, 2020

DOl: https://doi.org/10.21203/rs.3.rs-16306/v1

License: (c) (i) This work is licensed under a Creative Commons Attribution 4.0 International License.

Read Full License 


\section{Abstract}

Background Unresolved atelectasis occurs in some children with refractory mycoplasma pneumoniae pneumonia (RMPP). The aim was to analyze factors predicting unresolved atelectasis in RMPP and the impact of corticosteroids and bronchoscopy lavage therapy (BLT) on developing atelectasis.

Methods We retrospectively analyzed data for 230 pediatric RMPP from January 2013 to June 2017 in Beijing Children's Hospital. In this study, we diagnosed RMPP when patients' clinical and radiological findings deteriorated after 7 days of macrolide therapy, peripheral blood C-reactive protein (CRP) was higher than $40 \mathrm{mg} / \mathrm{L}$, and chest imaging showed consolidation with high density $>1 / 2$ pulmonary lobe. We divided patients into two groups according to the presence/absence of atelectasis on chest imaging after a 6-month follow-up. We calculated the predictive value of fever duration, levels of CRP and lactate dehydrogenase (LDH), and the size of lobe consolidation, regarding atelectasis. Additionally, we compared the starting time and dosage of corticosteroids and the starting time of BLT between the two groups.

Results Ninety-five patients developed atelectasis (atelectasis group/group A), and 135 patients did not (non-atelectasis group/group NA). Chest imaging showed $>2 / 3$ pulmonary lobe consolidation in $93.7 \%$ of patients in group A and $54.1 \%$ of patients in group NA. Multiple logistic regression analysis showed that fever duration, CRP and LDH levels, and lobe consolidation were related to developing atelectasis. Areas under the curve revealed that CRP $\geq 137 \mathrm{mg} / \mathrm{L}$ had $82.11 \%$ sensitivity and $80.07 \%$ specificity, and $\mathrm{LDH} \geq 471 \mathrm{IU} / \mathrm{L}$ had $62.65 \%$ sensitivity and $60.31 \%$ specificity to predict atelectasis. Fewer patients receiving corticosteroids and BLT within 10 days after illness onset developed atelectasis.

Conclusions Fever duration>10 days, CRP and LDH levels, and lobe consolidation are risk factors for developing atelectasis in RMPP. CRP $\geq 137 \mathrm{mg} / \mathrm{L}, \mathrm{LDH} \geq 471 \mathrm{IU} / \mathrm{L}$, and $>2 / 3$ pulmonary lobe consolidation were significant predictors of atelectasis, which can aid in early recognition. Corticosteroid administration and subsequent BLT within 10 days of the disease onset, and increased corticosteroid dosage may help reduce the incidence of atelectasis in these RMPP patients.

\section{Background}

Mycoplasma pneumoniae (MP) pneumonia (MPP) among children is significant for public health, which is traditionally described as mild and self-limited; however, more and more refractory, severe, fulminant or even fatal cases of MPP have been reported particularly in Eastern Asia in recent years ${ }^{[1-4]}$. MPP may deteriorate clinically and radiologically despite appropriate macrolide therapy, which defines refractory MPP (RMPP) $[2,4-6]$.

RMPP was not only difficult to cure and some of them may also develop sequelae such as unresolved atelectasis and bronchiectasis due to airway obliterans (AO), which will cause recurrent infection and then affect their quality of life ${ }^{[2,7]}$. Some of RMPP sequelae patients finally receive lobectomy. However, 
there have been only a few reported cases of MPP-associated $A O^{[7,8]}$, and the data on $A O$ caused by RMPP in large population are scarce.

Although atelectasis may gradually improve, it cannot be completely absorbed and finally develop bronchiectasis and bronchial occlusion in some RMPP patients ${ }^{[7]}$. Therefore, preventing unresolved atelectasis due to AO in RMPP should be the main goal of therapy. Early identification of the risk factors and implementing effective and timely treatment are important to achieve this goal. In this study, we analyzed the factors predicting unresolved atelectasis due to $A O$ and the effect of corticosteroids and subsequent bronchoscopy lavage therapy (BLT) on preventing unresolved atelectasis in RMPP.

\section{Methods}

\section{Study Population}

A total of 230 children with RMPP were recruited from January 2013 to June 2017 from the Respiratory Disease Department II, Beijing Children's Hospital affiliated to Capital Medical University, National Centre for Children's Health, the largest Children's Hospital in China. All patients were previously treated for at least 3 days at other hospitals or our outpatient clinic before admission to our inpatient department. In general, most RMPP patients could be cured in their local hospitals. However, in this study, most patients were from all over the countries and were referred to our department because of the treatment difficulty, so RMPP was more serious than those at other hospitals in China.

Using chest X-ray or computed tomography (CT) after a 6-month follow-up, 95 patients developed unresolved atelectasis (atelectasis group/group A, Fig.1) and 135 did not (non-atelectasis group/group NA). All the 95 patients with unresolved atelectasis had intraluminal obliterans confirmed on bronchoscopy on the late stage (Fig.2).

Diagnosis of MPP was performed according to the guidelines of the Chinese Medical Association ${ }^{[9]}$ : (1) clinical presentation (fever, cough); (2) chest imaging with infiltrates; (3) Serum anti-MP IgM titer $\geq 1: 320$ (double testing) or four-fold or greater rising titer in acute and convalescent serum specimens. In this study, we diagnosed RMPP when patients' clinical and radiological findings deteriorated after 7 days of macrolide therapy, and peripheral blood C-reactive protein (CRP) was higher than $40 \mathrm{mg} / \mathrm{L}^{[2,6]}$. Among them, patients with $>1 / 2$ pulmonary lobe consolidation on chest imaging were enrolled in this study.

Patients with congenital heart disease, hereditary metabolic diseases, neurological disorders, bronchopulmonary dysplasia, immunodeficiency, or concurrent bacterial and/or viral infections were excluded.

\section{Ethics}

Written informed consent was obtained from caregivers of all children to allow their clinical records to be used in this study. The study was conducted in accordance with the Declaration of Helsinki and was 
approved by the Ethics Committee of Beijing Children's Hospital affiliated Capital Medical University, National Center for Children's Health (IRB number: 2019-k-167). BLT in this study was based on the following standards: (1) Potential risk of the minor discomfort was minimized by utilizing only welltrained personnel for the procedures; (2) Written informed consent was provided to the parents or the guardians of children; (3) There are no contraindications about BLT in all patients. It is worth noting that the body temperature returned to normal in all patients when BLT was performed. Therefore most patients received BLT after being treated with corticosteroids. (4) All patients received BLT, because of the consolidation with high density $>1 / 2$ pulmonary lobe, and a critical method in detecting etiological agents of bronchoalveolar lavage fluid (BALF), according to the guideline in China ${ }^{[9,10] ;}$ (5) The participants and their parents or their guardians had the right to decide whether or not to participate; (6) The benefits to the patients and society could be critical to guide rational therapy and prevent atelectasis in pediatric RMPP.

\section{Fiberoptic bronchoscopy and BALF collection}

Fiberoptic bronchoscopy and BALF Collection were performed as previously reported ${ }^{[2]}$. The BALF samples $(2 \mathrm{~mL})$ from each patient were used for traditional bacterial culture and respiratory virus detection. MP gene detection was applied in 39 patients of Group A and 46 patients of Group NA.

\section{Statistical Analysis}

\section{Methods}

We retrospectively analysed fever duration, the highest serum CRP and lactate dehydrogenase (LDH) levels, chest X-ray or CT 6-10 days after illness onset, and dynamic bronchoscopic findings. We found that peak levels of CRP and LDH, and the most serious findings on chest X-ray or CT, occurred 6-10 days after illness onset. Therefore, we evaluated CRP and LDH levels and chest $X$-ray or CT findings 6-10 days after illness onset. We also retrospectively analysed the starting time and dose of corticosteroids and the starting time of BLT.

\section{Statistical methods}

We used SPSS software version 22 (IBM Inc., Armonk, NY) for all statistical analyses. The measurement data were normally distributed. The results were expressed as the mean \pm standard deviation and were analysed using the independent samples t-test. Numerical data were expressed as the rate or constituent ratio and were analysed using the chi-squared test. $\mathrm{P}<0.05$ was considered statistically significant. We used multivariate logistic regression analysis to determine the factors influencing the incidence of atelectasis in the groups A and NA. Significant differences in parameters between the two groups were considered independent risk factors. We used receiver operating characteristic (ROC) curve analysis as the gold standard to evaluate the development of atelectasis, and we used the area under the ROC curve (AUC) to assess the accuracy of the diagnostic test. The AUC range was $0.5-1$, with AUC $=0.5$ indicating zero diagnostic accuracy, AUC $>0.5$ and $<0.7$ indicating low diagnostic accuracy, AUC $>0.7$ and $<0.9$ 
indicating moderate diagnostic accuracy, AUC $>0.9$ and $<1$ indicating high diagnostic accuracy, and AUC $=1$ indicating diagnostic accuracy. The maximum sensitivity and specificity of the ROC curve closest to the left upper corner were considered the critical values for predicting atelectasis.

\section{Results}

\section{Patients' clinical characteristics}

Patients' mean age was $7.09 \pm 2.79$ years with a male/female ratio of $46: 49$ in group $A$ and $6.82 \pm 2.73$ years with a male/female ratio of 69:66 in group NA. No significant difference was found for the male/female ratio and age between the two groups. All patients presented with persistent high fever and cough in acute stage. Fever duration was significantly longer in group A than in group NA (Table 1).

\section{Laboratory examination}

\section{MP detection in BALF by PCR}

PCR for MP gene detection was positive in all of the 39 patients of Group A and 46 patients of Group NA. Genotype 2 was detected in 3 patients of Group A and 4 patients of Group NA. Genotype 1 was detected in the other patients. Only two strains (genotype 1 in Group A and genotype 2 in Group NA) were susceptible to the macrolide. The other strains were macrolide-resistant and carried the A2063G mutation.

\section{Peak CRP level}

The normal range for CRP in our laboratory is $<8 \mathrm{mg} / \mathrm{L}$. The peak CRP level was higher in group $A$ than that in group NA. CRP levels in $83.2 \%$ of patients in group A were $>130 \mathrm{mg} / \mathrm{L}$ vs. $22.2 \%$ in group NA. CRP levels in $61.1 \%$ of patients in group A were $>160 \mathrm{mg} / \mathrm{L}$ vs. $7.4 \%$ in group NA. The proportion of patients with $\mathrm{CRP}>200 \mathrm{mg} / \mathrm{L}$ was $29.5 \%$ in group $\mathrm{A}$ and no one in group NA (Table1).

\section{Peak LDH level}

The peak LDH level was higher in group $A$ at $729.8 \mathrm{IU} / \mathrm{L}$ vs. group NA at $486.2 \mathrm{IU} / \mathrm{L}$. The proportion of patients with $\mathrm{LDH}>500 \mathrm{IU} / \mathrm{L}$ was $72.6 \%$ in group $A$ and $36.3 \%$ in group NA (Table 1 ).

\section{Radiographic findings}

In group A, chest imaging showed consolidation with high density $>2 / 3$ pulmonary lobe in $93.7 \%$ of patients (Fig. 3), with mild to marked pleural effusion in $76.8 \%$ of these patients (Table 1). Additionally, $35.8 \%(34 / 95)$ of these patients had necrosis in the consolidated area after 2 weeks of illness onset.

In group NA, chest imaging showed consolidation with high density $>2 / 3$ pulmonary lobe in $54.1 \%$ of patients, with mild to moderate pleural effusion in $43.7 \%$ of these patients (Table 1 ). 
There was a significant difference in the proportion of patients with $>2 / 3$ pulmonary lobe consolidation and pleural effusion between the two groups, which suggested $>2 / 3$ lobe consolidation was an important predicting factor of atelectasis.

\section{Bronchoscopy findings}

Mucus plugs or airway cast formation and mucosal necrosis was found in both groups. $85.3 \%$ of patients had mucus plugs completely obstructing the lumen in group $A$ and $70.5 \%$ had mucosal necrosis vs. $14.8 \%$ and $6.7 \%$, respectively, in group NA (Table 1, Fig.4).

\section{Factors predicting unresolved atelectasis}

As shown in Table 1, fever duration, CRP and LDH levels, and the proportions of patients with 2/3 lobe consolidation, pleural effusion, and mucous plugs and mucosal necrosis differed between the two groups. Because fever duration and CRP and LDH levels can be quantified, we analyzed the association between CRP, LDH levels, and fever duration, and atelectasis. Multiple regression analysis revealed that CRP and LDH levels, and fever duration were associated with atelectasis [odds ratio (OR): 1.034, 1.002, and 1.121 , respectively) (Table 2 ).

Furthermore, because peak CRP and LDH levels, as two statistically-significant independent factors, are easy to obtain in clinical practice, we performed a ROC curve analysis using both CRP level and LDH level as predictors of atelectasis. The AUC for CRP level indicated that CRP level had a good diagnostic value (Fig. 5, Table 3).

\section{Starting time of glucocorticoids and bronchoscopy lavage therapy in each group}

As discussed, we found that the highest CRP level and the most serious lung consolidation occurred within 6-10 days after illness onset; mucus plugs and necrosis also appeared during this period. These findings suggested that the optimal time to start glucocorticoids and subsequent BLT was 6-10 days after illness onset. Clinically, we observed that patients were more likely to develop obliterans bronchitis/bronchiolitis if glucocorticoids and BLT were started 14 days after illness onset. In addition, the earliest staring time of glucocorticoid was 6 days after illness onset in this study. Therefore, we divided the starting time of glucocorticoids and BLT into three groups: $6-10$ days, 11-14 days, and after 14 days. Comparisons between the two groups (A and NA) for the starting time of glucocorticoids and BLT are shown in Table 4 and Table 5.

These results suggested that starting methylprednisolone and subsequent BLT within 14 days of illness onset, especially starting at 6-10 days, could reduce the incidence of atelectasis.

\section{Maximum glucocorticoid dose in each group}

Our results showed that starting treatment with methylprednisolone and subsequent BLT within 14 days of illness onset can prevent atelectasis. 
In fact, in group A, 21 patients received both methylprednisolone and BLT within 14 days after illness, but atelectasis still occurred (Table 5, Table 6). Additionally, CRP levels were higher than $130 \mathrm{mg} / \mathrm{L}$ in all these 21 patients (Table 6). 10 patients whose CRP levels were $>200 \mathrm{mg} / \mathrm{L}$ received $6-20 \mathrm{mg} / \mathrm{kg} . \mathrm{d}$ of methylprednisolone after 7 days of illness onset (Table 6).

In contrast, 71 patients received both methylprednisolone and BLT within 14 days in group NA, and 10 patients had CRP levels of 160-200 mg/L; the remaining patients had levels $<160 \mathrm{mg} / \mathrm{L}$. Ten patients in group NA received 6-20 mg/kg.d of methylprednisolone, while 5 patients with the same CRP levels in group A received 2-6 mg/kg.d of methylprednisolone (Table 6).

Despite the lack of statistical analysis because of the small sample size, differences between the two groups suggest that the dose of glucocorticoid may have an effect on the development of atelectasis.

In addition, although both methylprednisolone and BLT were started after 14 days in 16 patients in group NA, these patients did not develop atelectasis (Table 4, Table 6). CRP values in each of these 16 children were $<130 \mathrm{mg} / \mathrm{L}$, while CRP values in 37 patients in group A with CRP $>130 \mathrm{mg} / \mathrm{L}$ received $2 \mathrm{mg} / \mathrm{kg} . \mathrm{d}$ of methylprednisolone, further supporting the hypothesis that atelectasis may be associated with excessive inflammatory response and the dose of methylprednisolone (Table 6).

Moreover, the other patients in groups A and NA received 2-4 mg/kg.d of methylprednisolone.

\section{Anti-MP therapy}

All patients were treated with macrolides. In addition, moxifloxacin was also added to the treatment course in 16 patients and 4 patients in groups $A$ and NA, respectively.

\section{Discussion}

MPP is a common community-acquired pneumonia in children, and RMPP is a well-known condition particularly in North China. RMPP can lead to unresolved atelectasis, and unresolved atelectasis is associated with $A O$ which has been reported mainly in our previous studies ${ }^{[2,7,8]}$. To our knowledge, ours is the first study to determine the factors predicting atelectasis in pediatric RMPP and to evaluate the influence of methylprednisolone and BLT on atelectasis development in children with RMPP.

Macrolide-resistant (MR)-MP strains are becoming increasingly common in Eastern Asia, and have been reported at a rate of $>90 \%$ in many areas in China including our previous publications ${ }^{[11,12]}$. In our present study and in China, type 1 was the main genotype ${ }^{[11]}$ and the total MR-MP rate was high up to $97.7 \%$ (83/85). Macrolides are still used as first-line agents at high frequencies in countries with a high burden of MR-MP strains, such as China and Japan, mainly because of their low minimum inhibitory concentrations against the MP, anti-inflammatory effect and low toxicity in children. Yoon et al. reported macrolide treatment for macrolide-susceptible MP pneumonia did not contribute to significant clinical improvement compared to no antimicrobial treatment ${ }^{[13]}$. Yang et al. reported corticosteroid therapy 
might prevent disease progression, and anti-MP-antibiotics may have limited effects on MPP ${ }^{[1]}$. We agree with the above opinions that even timely macrolide-susceptible antibiotics such as moxifloxacin couldn't prevent disease progression through clinical experience ${ }^{[14]}$, so we didn't highly focus on the anti-MP treatment in this study.

Our results suggested that patients who developed atelectasis had longer fever duration, higher serum CRP and LDH levels, larger area of lung consolidation ( $>2 / 3$ lobe), and more frequent intraluminal obstruction with mucus plugs and mucosal necrotic exudate. Serum CRP and LDH are important inflammatory markers especially in pediatric RMPP $[2,6,15-18]$. Longer fever duration, higher CRP and LDH levels in group A suggested more excessive inflammatory response, which further suggested these patients need timely higher dose corticosteroid treatment. In our study, the peak value of CRP $>137 \mathrm{mg} / \mathrm{L}$ within 6-10 days after illness was a better predictive atelectasis marker in pediatric RMPP than LDH $\geq$ $471 \mathrm{IU} / \mathrm{L}$.

Host hyperimmune reaction against MP infection is responsible for lung cell injury in pediatric RMPP[1923]. The immune reaction of the host before the peak of inflammation (proinflammatory cytokines may be involved in this stage) may be involved in pulmonary tissue cell injury, and immune reaction after the peak of inflammation may be involved in pulmonary tissue cell repair (anti-inflammatory cytokines may be involved in the convalescent stage), and the intensity of systemic inflammation during this process is reflected in laboratory parameters such as CRP, LDH, and immune proteins such as cytokines and chemokines ${ }^{[23]}$. Thus, early control of this process is critical in treatment of MPP, which will reduce the disease progression.

Some studies reported that corticosteroids had positive effects on fever and consolidation in MPP ${ }^{[1,3-5 \text {, }}$ ${ }^{24]}$, but the development of unresolved atelectasis was not followed in these studies, although these patients showed temperature recover after treatment. In addition, the influence of the starting time and dosage of corticosteroids on the development of unresolved atelectasis has not been reported. Moreover, these previous studies didn't talk about BLT and bronchoscopy findings.

In this study, we found that starting methylprednisolone within 14 days of illness onset, especially within 6-10 days, reduced the occurrence of atelectasis. Additionally, 6-10 days coincided with the peak CRP level, airway hypersecretion such as mucus plugs and lobe consolidation, indicating that the inflammatory response was the most serious during this period. In previous reports, doses of $1-$ $2 \mathrm{mg} / \mathrm{kg} . \mathrm{d}$ prednisone and $30 \mathrm{mg} / \mathrm{kg}$.d methylprednisolone had positive effects on fever and improved or resolved lung consolidation ${ }^{[4,5,25]}$. Our results showed that $2 \mathrm{mg} / \mathrm{kg}$.d methylprednisolone was ideal for preventing unresolved atelectasis in most of the patients with CRP values $<130 \mathrm{mg} / \mathrm{L}$ and $<2 / 3$ lobe consolidation. We have reported that increased CRP levels reflected an excessive inflammatory reaction than a mixed bacterial infection ${ }^{[2]}$, so in theory, higher CRP levels reflect stronger inflammatory reactions. In addition, traditional bacterial culture of BALF was negative in all of our patients. Therefore, we hypothesized that the dose of methylprednisolone should increase with the degree of inflammation. 
Because most patients who developed atelectasis in our study had CRP levels $>130 \mathrm{mg} / \mathrm{L}$ and $>2 / 3$ lobe consolidation, doses of methylprednisolone higher than $2 \mathrm{mg} / \mathrm{kg} . \mathrm{d}$ even pluse therapy may be beneficial. Our results also suggested that higher methylprednisolone doses may reduce the frequency of atelectasis, supporting a dose of methylprednisolone based on the levels of inflammatory markers such as CRP, LDH and the degree of lobe consolidation. However, determining the guidelines for corticosteroid dose selection requires prospective studies.

To our knowledge, only a few studies mainly Chinese studies have reported of the effective and welltolerated BLT and bronchoscopy findings such as mucus plugs on the early stage of RMPP $[14,17,26-28]$. It has been reported that airway hypersecretion, epithelial desquamation, and remodeling cause $A O$ in a human airway epithelium model with M. pneumoniae infection ${ }^{[29]}$. MP induces airway mucus hypersecretion by modulating the STAT/EGFR-FOXA2 signaling pathways ${ }^{[30]}$. Our results showed that the bronchial lumen was obstructed by mucus plugs even bronchial cast in patients who developed atelectasis, and that $\mathrm{AO}$ developed quickly, if the mucus plug was not removed (data not shown). However, once AO develops, atelectasis cannot be reversed ${ }^{[2,8]}$; therefore, timely BLT to eliminate the mucus plugs is reasonable to prevent atelectasis on the early stage in pediatric RMPP. In addition, some patients had to receive bronchoscopic interventional therapy such as balloon dilatation because of atelectasis caused by $\mathrm{AO}$ or airway stenosis on the late stage in group A. This study showed that BLT within 6-10 days of illness onset can decrease the chances of developing atelectasis, and 6-10 days is consistent with the strongest inflammatory response period. However, in group A, 10 patients whose CRP levels were $>200 \mathrm{mg} / \mathrm{L}$ received $6-20 \mathrm{mg} / \mathrm{kg}$.d of methylprednisolone after 7 days of illness onset (within 14 days) (Table 6), suggesting that other factors may affect the development of atelectasis; further study is needed.

The limitations of this study include its retrospective design and that the number of patients who received 10-30 mg/kg.d methylprednisolone was insufficient for a stratified analysis. In addition, a small number of patients with $\mathrm{CRP}<130 \mathrm{mg} / \mathrm{L}$ developed atelectasis, and we did not investigate the cause. However, our results provide helpful guidelines to determine patients with a high risk of developing atelectasis and to prevent atelectasis in RMPP. The disease duration was more than 5 days before admission to our department in all patients, therefore corticosteroid could not be administered earlier.

\section{Conclusions}

Long fever duration, high levels of inflammatory markers such as CRP and LDH, and large area of lobe consolidation suggested an airway hypersecretion such as airway mucus plugs. Fever duration $>10$ days, $\mathrm{CRP} \geq 137 \mathrm{mg} / \mathrm{L}, \mathrm{LDH} \geq 471 \mathrm{IU} / \mathrm{L},>2 / 3$ pulmonary lobe consolidation were significant predictors of atelectasis in pediatric RMPP, which can aid in early recognition. Both corticosteroid administration and subsequent BLT within 10 days of the onset of disease, and increased corticosteroid dosage may help reduce the incidence of atelectasis in these RMPP children. 


\section{Abbreviations}

AO: airway obliterans; BALF: bronchoalveolar lavage fluid; BLT: bronchoscopy lavage therapy; CRP: C reactive protein; LDH: lactate dehydrogenase; MP: Mycoplasma pneumoniae, MPP: Mycoplasma pneumoniae pneumonia; RMPP: refractory MPP.

\section{Declarations}

\section{Ethics approval and consent to participate}

All procedures performed in studies involving human participants were in accordance with the ethical standards of the Ethics Committee of Beijing Children's Hospital affiliated to Capital Medical University, National Center for Children's Health (No. 2017-23) and with the 1964 Helsinki declaration and its later amendments or comparable ethical standards. Participant consent was written by the guardian of all the patients and informed consent forms were archived.

\section{Consent for publication}

Not applicable.

\section{Availability of data and materials}

The data and materials are not available because of hospital regulation.

\section{Competing interests}

The authors declare that they have no competing interests.

\section{Funding}

This work was supported by Beijing Municipal and Commission Health and Family Planning (Grant No. 2015-3-076). The funding body had no role in the design of the study and collection, analysis, and interpretation of data and in writing the manuscript.

\section{Authors' contributions}

$\mathrm{J}-\mathrm{H} \mathrm{L}$ and $\mathrm{J}-\mathrm{R} \mathrm{L}$ wrote the main manuscript text; BW, $\mathrm{YL}, \mathrm{CZ}, \mathrm{FZ}$, and $\mathrm{JZ}$ collected and analyzed clinical data; FZ and JZ performed MP related testing and analyzed experimental data; J-H L, J-R L, and SZ are to take responsibility for study design. All authors reviewed the manuscript. All authors read and approved the final manuscript.

\section{Acknowledgments}

We thank Jane Charbonneau, DVM, from Liwen Bianji, Edanz Group China (www.liwenbianji.cn/ac), for editing the English text of a draft of this manuscript. We also thank all of the patients and their families for their kind cooperation.

\section{Author details}


${ }^{1}$ Department of Respiratory Medicine II, Beijing Children's Hospital, National Center for Children's Health, Capital Medical University, N0.56, Nanlishi Road 56, Xicheng District, Beijing, China. ${ }^{2}$ Department of Pediatrics, Maternal and Child Health Care Hospital of Shunyi District, NO.1, Shunkang Road, Shunyi District, Beijing, China. ${ }^{3}$ Department of Radiology, Beijing Children's Hospital, National Centre for Children's Health, Capital Medical University, NO.56, Nan Li Shi Road, Beijing, China. ${ }^{4}$ Health Care Department of Pediatrics, Maternal and Child Health Care Hospital of Shunyi District, NO.1, Shunkang Road, Shunyi District, Beijing, China. ${ }^{5}$ National Institute for Communicable Disease Control and Prevention, Chinese Center for Disease Control and Prevention; State Key Laboratory of Infectious Disease Prevention and Control, NO.155, Changbai Road, Changping District, Beijing, China.

\section{References}

[1] Yang EA, Kang HM, Rhim JW, Kang JH, Lee KY. Early Corticosteroid Therapy for Mycoplasma pneumoniae Pneumonia Irrespective of Used Antibiotics in Children. J Clin Med. 2019; 8(5).

[2] Liu JR, Lu J, Dong F, et al. Low Bacterial Co-infection Invalidates the Early Use of Non-antiMycoplasma pneumoniae Antibiotics in Pediatric Refractory Mycoplasma pneumoniae Pneumonia Patients. Front Pediatr. 2018; 6: 296.

[3] You SY, Jwa HJ, Yang EA, Kil HR, Lee JH. Effects of Methylprednisolone Pulse Therapy on Refractory Mycoplasma pneumoniae Pneumonia in Children. Allergy Asthma Immunol Res. 2014; 6(1): $22-6$.

[4] Tamura A, Matsubara K, Tanaka T, Nigami H, Yura K, Fukaya T. Methylprednisolone pulse therapy for refractory Mycoplasma pneumoniae pneumonia in children. J Infect. 2008; 57(3): 223-8.

[5] Lee KY, Lee HS, Hong JH, et al. Role of prednisolone treatment in severe Mycoplasma pneumoniae pneumonia in children. Pediatr Pulmonol. 2006; 41(3): 263-8.

[6] Liu JR, Peng Y, Yang HM, Li HM, Zhao SY, Jiang ZF. [Clinical characteristics and predictive factors of refractory Mycoplasma pneumoniae pneumonia]. Zhonghua Er Ke Za Zhi. 2012; 50(12): 915-8.

[7] Leong MA, Nachajon R, Ruchelli E, Allen JL. Bronchitis obliterans due to Mycoplasma pneumonia. Pediatr Pulmonol. 1997; 23(5): 375-81.

[8] Zhao C, Liu J, Yang H, Xiang L, Zhao S. Mycoplasma pneumoniae-Associated Bronchiolitis Obliterans Following Acute Bronchiolitis. Sci Rep. 2017; 7(1): 8478.

[9] Subspecialty Group of Respiratory Diseases, The Society of Pediatrics, Association CM, Editorial Board, Chinese Journal of Pediatrics. [Guidelines for management of community acquired pneumonia in children (the revised edition of 2013) (I)]. Zhonghua Er Ke Za Zhi. 2013; 51(10): 745-52. 
[10] Experts Group of Pediatric Respiratory Endoscopy TESCoNHC. Guideline of pediatric flexible bronchoscopy in China. Chin J Appl Clin Pediatr. 2018; 33(13): 983-989.

[11] Zhao F, Li J, Liu J, et al. Antimicrobial susceptibility and molecular characteristics of Mycoplasma pneumoniae isolates across different regions of China. Antimicrob Resist Infect Control. 2019; 8: 143.

[12] Zhao F, Liu J, Shi W, et al. Antimicrobial susceptibility and genotyping of Mycoplasma pneumoniae isolates in Beijing, China, from 2014 to 2016. Antimicrob Resist Infect Control. 2019; 8: 18.

[13] Yoon IA, Hong KB, Lee HJ, et al. Radiologic findings as a determinant and no effect of macrolide resistance on clinical course of Mycoplasma pneumoniae pneumonia. BMC Infect Dis. 2017; 17(1): 402.

[14] Liu J, Zhao F, Lu J, et al. High Mycoplasma pneumoniae loads and persistent long-term Mycoplasma pneumoniae DNA in lower airway associated with severity of pediatric Mycoplasma pneumoniae pneumonia. BMC Infect Dis. 2019; 19(1): 1045.

[15] Chen L, Liu J, Zhao S, Yang Y, Wu J. [Clinical features and treatment of refractory Mycoplasma pneumoniae pneumonia unresponded to conventional dose methylprednisolone in children]. Zhonghua Er Ke Za Zhi. 2014; 52(3): 172-6.

[16] Yu JL, Song QF, Xie ZW, et al. iTRAQ-based Quantitative Proteomics Study in Patients with Refractory Mycoplasma pneumoniae Pneumonia. Jpn J Infect Dis. 2017; 70(5): 571-578.

[17] Yan Y, Wei Y, Jiang W, Hao C. The clinical characteristics of corticosteroid-resistant refractory Mycoplasma Pneumoniae pneumonia in children. Sci Rep. 2016; 6: 39929.

[18] Choi YJ, Jeon JH, Oh JW. Critical combination of initial markers for predicting refractory Mycoplasma pneumoniae pneumonia in children: a case control study. Respir Res. 2019; 20(1): 193.

[19] Tanaka H, Narita M, Teramoto S, et al. Role of interleukin-18 and T-helper type 1 cytokines in the development of Mycoplasma pneumoniae pneumonia in adults. Chest. 2002; 121(5): 1493-7.

[20] Waites KB, Talkington DF. Mycoplasma pneumoniae and its role as a human pathogen. Clin Microbiol Rev. 2004; 17(4): 697-728, table of contents.

[21] Youn YS, Lee KY. Mycoplasma pneumoniae pneumonia in children. Korean J Pediatr. 2012; 55(2): 42-7.

[22] Sekine $\mathrm{H}$, Taguchi $\mathrm{H}$, Watanabe $\mathrm{H}$, et al. Immunological analysis and pathological examination of gnotobiotic mice monoassociated with Mycoplasma pneumoniae. J Med Microbiol. 2009; 58(Pt 6): 697705.

[23] Lee KY. Pneumonia, Acute Respiratory Distress Syndrome, and Early Immune-Modulator Therapy. Int J Mol Sci. 2017; 18(2). 
[24] Youn YS, Lee KY, Hwang JY, et al. Difference of clinical features in childhood Mycoplasma pneumoniae pneumonia. BMC Pediatr. 2010; 10: 48.

[25] Luo Z, Luo J, Liu E, et al. Effects of prednisolone on refractory mycoplasma pneumoniae pneumonia in children. Pediatr Pulmonol. 2014; 49(4): 377-80.

[26] Huang L, Huang X, Jiang W, Zhang R, Yan Y, Huang L. Independent predictors for longer radiographic resolution in patients with refractory Mycoplasma pneumoniae pneumonia: a prospective cohort study. BMJ Open. 2018; 8(12): e023719.

[27] Wang L, Lu S, Feng Z, et al. The early examination of combined serum and imaging data under flexible fiberoptic bronchoscopy as a novel predictor for refractory Mycoplasma pneumoniae pneumonia diagnosis. Medicine (Baltimore). 2017; 96(50): e9364.

[28] Zhang $Y$, Chen $Y$, Chen Z, et al. Effects of bronchoalveolar lavage on refractory Mycoplasma pneumoniae pneumonia. Respir Care. 2014; 59(9): 1433-9.

[29] Prince OA, Krunkosky TM, Sheppard ES, Krause DC. Modelling persistent Mycoplasma pneumoniae infection of human airway epithelium. Cell Microbiol. 2018; 20(3).

[30] Hao Y, Kuang Z, Jing J, et al. Mycoplasma pneumoniae modulates STAT3-STAT6/EGFR-FOXA2 signaling to induce overexpression of airway mucins. Infect Immun. 2014; 82(12): 5246-55.

\section{Tables}

Table 1. Single factors associated with atelectasis

\begin{tabular}{|c|c|c|c|c|}
\hline Project & Group A $(\mathrm{n}=95)$ & Group NA $(n=135)$ & Statistic & P (bilateral) \\
\hline Fever duration (d) & $16.97 \pm 8.716$ & $11.58 \pm 3.22$ & $t=5.758$ & 0.000 \\
\hline $\mathrm{CRP}(\mathrm{mg} / \mathrm{L})$ & $193.12 \pm 59.37$ & $98.42 \pm 42.20$ & $t=13.352$ & 0.000 \\
\hline $\mathrm{CRP}<130 \mathrm{mg} / \mathrm{L}$ & $16.8 \%(16 / 95)$ & $77.8 \%(77 / 135)$ & $X^{2}=37.41$ & 0.000 \\
\hline $\mathrm{CRP}>130 \mathrm{mg} / \mathrm{L}$ & $83.2 \%(79 / 95)$ & $22.2 \%(30 / 135)$ & $X^{2}=83.05$ & 0.000 \\
\hline $\mathrm{CRP}>160 \mathrm{mg} / \mathrm{L}$ & $61.1 \%(58 / 95)$ & $7.4 \%(10 / 135)$ & $X^{2}=77.06$ & 0.000 \\
\hline $\mathrm{CRP}>200 \mathrm{mg} / \mathrm{L}$ & $29.5 \%(28 / 95)$ & $0 \%(0 / 135)$ & - & - \\
\hline LDH (IU/L) & $729.8(660.47-814.1)$ & $486.2(452.3-522.8)$ & $Z=-6.405$ & 0.000 \\
\hline $\mathrm{LDH}>500 \mathrm{IU} / \mathrm{L}$ & $72.6 \%(69 / 95)$ & $36.3 \%(49 / 135)$ & $X^{2}=29.47$ & 0.000 \\
\hline \multicolumn{5}{|l|}{ Chest imaging } \\
\hline$\geq 2 / 3$ lobe consolidation & $93.7 \%(89 / 95)$ & $54.1 \%(73 / 135)$ & $\mathrm{X}^{2}=14.64$ & 0.000 \\
\hline Pleural effusion & $76.8 \%(73 / 95)$ & $43.7 \%(59 / 135)$ & $X^{2}=25.04$ & 0.000 \\
\hline \multicolumn{5}{|l|}{ Broncoscopy findings } \\
\hline Mucus plug & $85.3 \%(81 / 95)$ & $14.8 \%(20 / 135)$ & $X^{2}=22.85$ & 0.000 \\
\hline Mucosal necrosis & $70.5 \%(67 / 95)$ & $6.7 \%(9 / 135)$ & $X^{2}=102.78$ & 0.000 \\
\hline
\end{tabular}


Table 2. Multiple logistic regression analysis

\begin{tabular}{cccc}
\hline Variable & OR & $95 \%$ CI & P value \\
\hline CRP & 1.033 & $1.022-1.044$ & 0.000 \\
LDH & 1.001 & $1.000-1.003$ & 0.044 \\
Fever duration & 1.200 & $1.014-1.419$ & 0.034 \\
\hline
\end{tabular}

OR, odds ratio; CI, confidence level

Table 3. Ability of CRP and LDH levels to predict atelectasis

\begin{tabular}{lllllll}
\hline Independent factor & Critical value & Sensitivity & Specificity & AUC & P & Youden index \\
\hline $\mathrm{CRP}$ & $137 \mathrm{mg} / \mathrm{L}$ & $82.11 \%$ & $80.74 \%$ & 0.893 & $<0.0001$ & 0.6285 \\
$\mathrm{LDH}$ & $471 \mathrm{IU} / \mathrm{L}$ & $62.65 \%$ & $60.31 \%$ & 0.627 & 0.0016 & 0.2296 \\
\hline
\end{tabular}

AUC, area under the receiver operating characteristic (ROC) curve; CRP, C-reactive protein; LDH, lactate dehydrogenase. The Pvalue is compared with the AUC value of the related independent factors and the reference value for the ROC curve (0.5). SI conversion factors: CRP, LDH.

Table 4 Starting time of methylprednisolone administration in each group

\begin{tabular}{lllll}
\hline Starting time & Group A (\%) & Group NA (\%) & $X^{2}$ & P (bilateral) \\
\hline 6 -10 days & $34.7 \%(33 / 95)$ & $57.0 \%(77 / 135)$ & & \\
$11-14$ days & $24.2 \%(23 / 95)$ & $31.1 \%(42 / 135)$ & 26.261 & 0.000 \\
$>14$ days & $41.1 \%(39 / 95)$ & $11.9 \%(16 / 135)$ & & \\
\hline
\end{tabular}

Note: 6 -10 days group compared with the $11-14$ days group $\left(\mathrm{X}^{2}=0.544 ; \mathrm{P}=0.461\right) ; 6-10$ days group compared with the $>14$ days group, $\left(\mathrm{X}^{2}=24.950,<0.0001\right) ; 11-14$ days group compared with the $>14$ days group $\left(\mathrm{X}^{2}=15.055,<0.0001\right)$.

Table 5 Starting time of bronchoscopic treatment in each group

\begin{tabular}{lllll}
\hline Starting time & Group A (\%) & Group NA (\%) & Statistic $\left(\mathrm{X}^{2}\right)$ & P (bilateral) \\
\hline 6-10 days & $4.2 \%(4 / 95)$ & $14.1 \%(19 / 135)$ & & \\
$11-14$ days & $17.9 \%(17 / 95)$ & $41.5 \%(56 / 135)$ & 5.908 & 0.000 \\
$>14$ days & $77.9 \%(74 / 95)$ & $44.4 \%(60 / 135)$ & & \\
\hline
\end{tabular}

Note: 6-10 days group compared with the 11-14 days group $\left(\mathrm{X}^{2}=0.356, \mathrm{P}=0.551\right)$; 6 -10 days group compared with the $>14$ days group $\left(\mathrm{X}^{2}=11.239, \mathrm{P}=0.001\right) ; 11-14$ days group compared with the $>14$ days group $\left(\mathrm{X}^{2}=16.564, \mathrm{P}=0.000\right)$.

Table 6 Maximum glucocorticoid dose in each group 


\begin{tabular}{|c|c|c|}
\hline Starting time & Group A (cases) & Group NA (cases) \\
\hline Within 14 days (both MP and BT) & $21(21 / 95,22.1 \%)$ & $71(71 / 135,52.6 \%)$ \\
\hline $\mathrm{CRP}<130 \mathrm{mg} / \mathrm{L}$ & 0 & 50 (MDOM: $2-4 \mathrm{mg} / \mathrm{kg} . \mathrm{d})$ \\
\hline CRP:130-160 mg/L & 6 (MDOM: 2-4mg/kg.d) & 11 (MDOM: 2-5mg/kg.d) \\
\hline CRP: $160-200 \mathrm{mg} / \mathrm{L}$ & 5 (MDOM: $2-6 m g / k g . d)$ & 10 (MDOM: $6-20 \mathrm{mg} / \mathrm{kg} . \mathrm{d}$ ) \\
\hline $\mathrm{CRP}>200 \mathrm{mg} / \mathrm{L}$ & 10 (MDOM:6-20mg/kg.d) & 0 \\
\hline After 14 days (both MP and BT) & $37(37 / 95,38.9 \%)$ & $16(16 / 135,11.9 \%)$ \\
\hline $\mathrm{CRP}<130 \mathrm{mg} / \mathrm{L}$ & 0 & 16 (MDOM: 2mg/kg.d) \\
\hline $\mathrm{CRP}>130 \mathrm{mg} / \mathrm{L}$ & 37 (MDOM: 2mg/kg.d) & 0 \\
\hline - (both MP and BT) & $\begin{array}{l}37(37 / 95,38.9 \%) \\
\text { (MDOM: } 2-4 \mathrm{mg} / \mathrm{kg} . \mathrm{d})\end{array}$ & $\begin{array}{l}48(48 / 135,35.6 \%) \\
\text { (MDOM: } 2-4 \mathrm{mg} / \mathrm{kg} . \mathrm{d})\end{array}$ \\
\hline
\end{tabular}

MDOM, Maximum dose of methylprednisolone; MP, methylprednisolone; BT, bronchoscopic treatment.

\section{Figures}

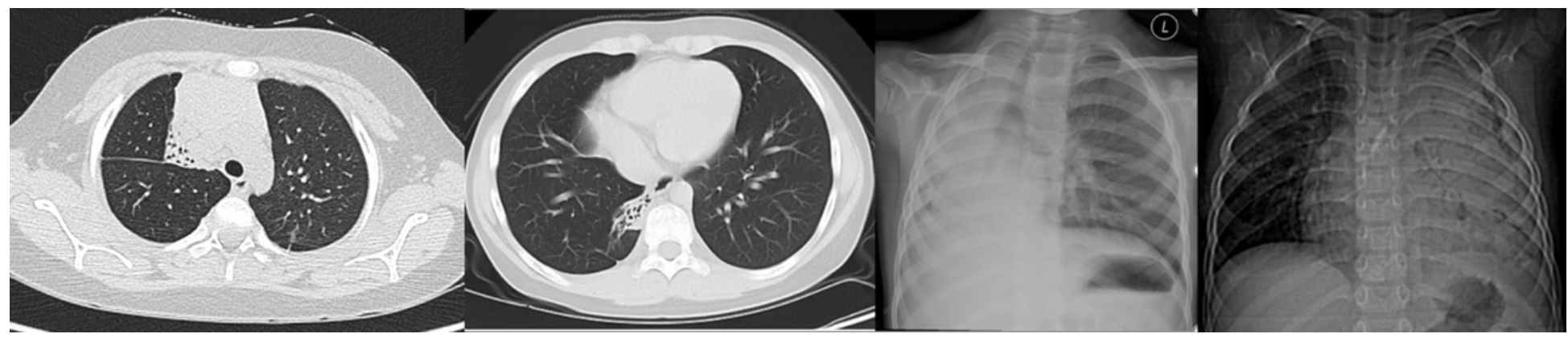

\section{Figure 1}

Chest imaging revealed localized atelectasis and even bronchiectasis after more than 1 year of illness onset.

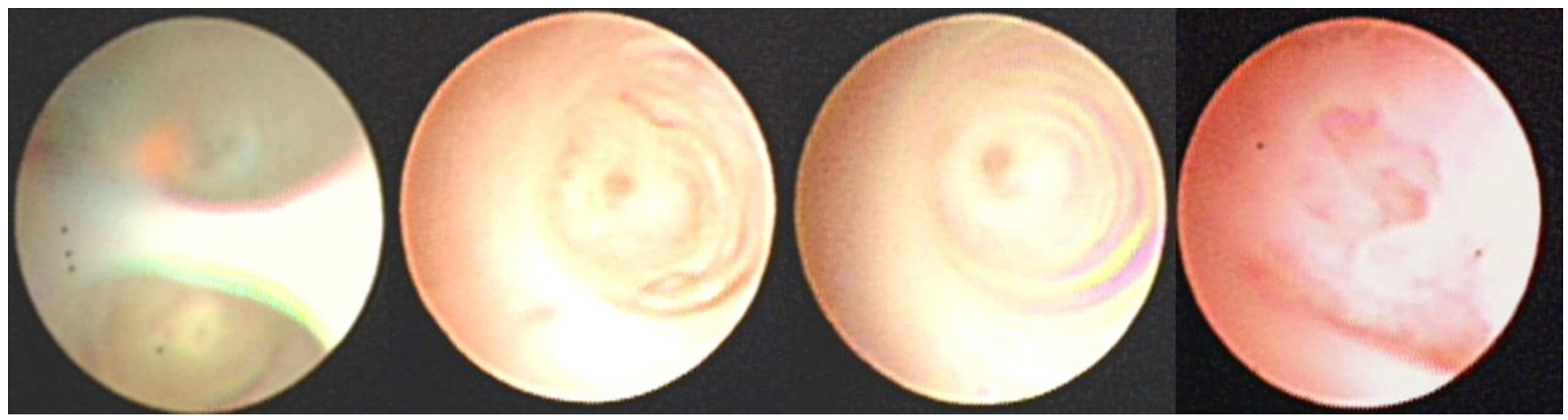

Figure 2

Fiberoptic bronchoscopy revealed the obliteration of the lumen of airway on the late stage. 


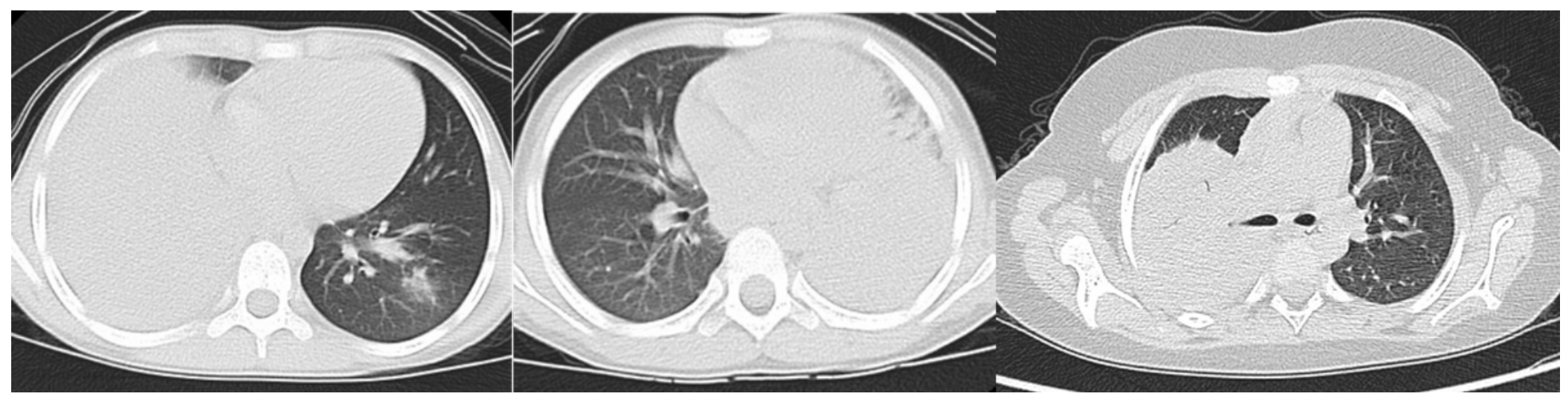

Figure 3

Chest imaging revealed high-density consolidation.

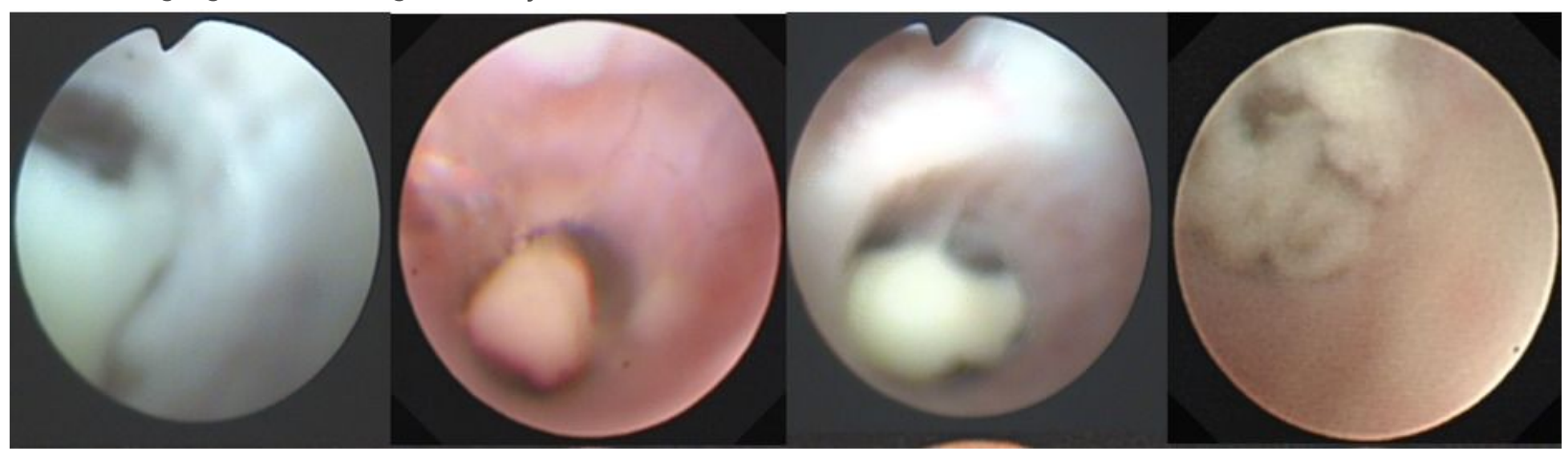

\section{Figure 4}

Fiberoptic bronchoscopy revealed mucus plug in the airway on the early stage.
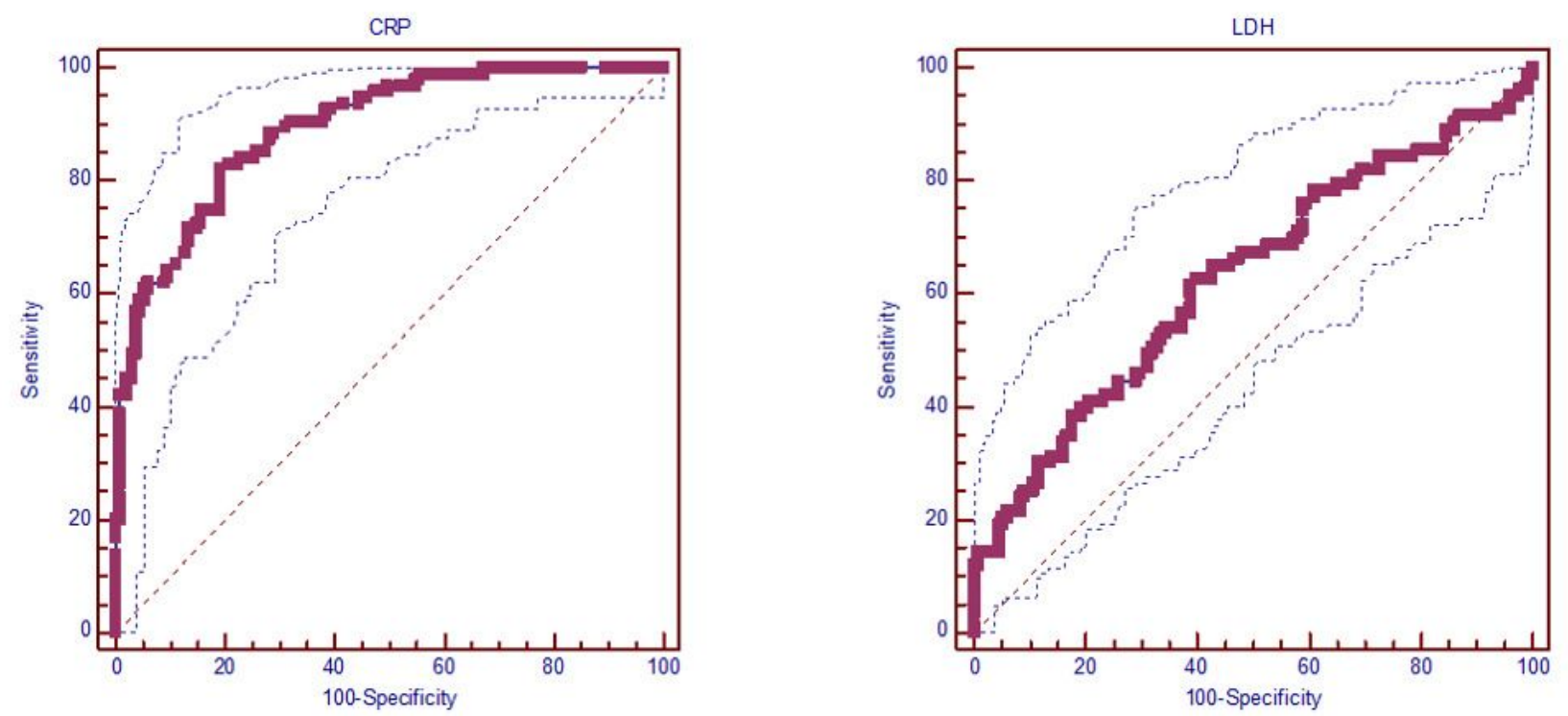

Figure 5 
ROC curve analysis about CRP and LDH.

Page 17/17 